\title{
Der Basler Chirurg und Rebell Johannes Fatio $1649-1691$
}

Von F. Rintelen

Etwa 90 Namen von Persönlichkeiten kann man auf Basels Straßenschildern lesen. Eine einzige Frau ist dabei: Mathilde Paravicini, Betreuerin kriegsgeschädigter Kinder, 1942 mit dem medizinischen Ehrendoktorat ausgezeichnet. An manche Ärzte-Mediziner - wird erinnert, an Paracelsus, Vesal, die Zwinger und Bernoulli, an K.G.Jung, Friedrich Miescher, Wilhelm His, an Socin, Rütimeyer, Albert Schweitzer und Karl Jaspers; nach Felix Platter heißt ein Spital, nach Gustav v. Bunge ein Brunnen; den ehemaligen Stuttgarter Regimentsmedicus Friedrich Schiller wird man kaum zu den Ärzten zählen. Eine kleine Seitenstraße im westlichen GroßBasel trägt den Namen Fatio. 1925 hat sie die Bezeichnung bekommen. Eine «Nomenklatur-Kommission» für Straßennamen gibt es in Basel seit 1947. Vorher war allein der Regierungsrat zuständig, der sich meist durch den jeweiligen Staatsarchivar beraten lie ${ }^{1,2}$. Man darf annehmen, da 3 sich 1925 der auch um die Universität so verdiente Regierungsrat Dr. Fritz Hauser für die späte Anerkennung des gegen arge Amtsmißbräuche der Basler regierenden Oberschicht 1691 schließlich erfolglos revoltierenden und hingerichteten Johann Fatio eingesetzt hat. Wer weiß heute noch - auch in Basel-über diesen ungewöhnlichen Mann Bescheid, der sich als Chirurg und Geburtshelfer in der Stadt so nützlich gemacht hat?

Die Fatio - oder Fazzy - stammen aus dem Eschental, dem Val d'Ossola, das 1516 nach der «ewigen Richtung» für die Eidgenossen verlorengegangen ist. Genaueres ist erst über einen Jean Fatio (1530-1597) bekannt ${ }^{3}$. Er hat als Protestant seine Heimat verlassen und ist zunächst in Vicosoprano, 1594 in Chiavenna eingebürgert worden. Auch dort war ein Bleiben für die Fatio nicht möglich. Der «Veltliner-Mord», der auch Cleven nicht verschonte, vom Juli 1620, veranlaßte Familien neuen Glaubens wie die Fatio zur Flucht. Johann Fatio, Sohn des Jean, geboren 1591, ist mit seiner Frau und zwei kleinen Söhnen, gleichzeitig mit Jenatsch den Mordgesellen entkommen, die im Veltlin und Bergell eine Bartholomäusnacht inszenieren wollten ${ }^{4}$. Mit Johann floh auch sein ältester Bruder Paul (1587-1656). Beide sind zunächst ins protestantische Zürich gelangt. Johann ist mit den Seinen über Wien schließlich nach Basel gekommen und Stammvater des Basler Zweiges der 
Fatio geworden. Paul, zuerst nach Vivis, dann nach Genf ausgewandert, wird Begründer der Viviser Linie der Fatiofamilie. Sein Sohn François (1622-1704) ist als Genfer Bürger ein begüterter Bankier geworden. Johann wird schon 1635 ins Basler Bürgerrecht aufgenommen - es galt Pest-Lücken zu schließen! Der gewiegte Kaufmann hat es bald zu Ansehen und Vermögen gebracht. 1659 ist er gestorben ${ }^{4}$. Im Kreuzgang des Münsters findet man seine Grabtafel. Von seinen fünf Söhnen verheiratete sich der älteste, Johann-Anton, mit Christine Henric-Petri, der Tochter eines angesehenen Juristen aus einer seit dem 15. Jahrhundert in Basel bekannten Buchdrukker-Familie. Johann-Anton ist der Vater des 1649 geborenen Johann Fatio, der uns hier beschäftigt. Der zweite Sohn Johannes, Baptist, ist nach kurzer Basler-Zeit, mit einer Waadtländerin verheiratet, nach Genf gezogen und dort eingebürgert worden. Er hat das Gut Duillier erworben; seine Familie nannte sich seither Fatio d'Duillier. Baptist ist 1709 gestorben. Entgegen den Angaben im Historisch-biographischen Lexikon der Schweiz ${ }^{3}$ und der Darstellung von Albert Huber ${ }^{4}$ stammen also die welschen Fatios aus zwei Familienzweigen. Ein Nachkomme des sogenannten Viviser Stammes, Pierre Fatio, docteur en droit, hat ein ähnliches Schicksal erfahren wie sein entfernter Basler Vetter Johann. Er ist als «chef du parti populaire» in einen Aufstand gegen die Genfer Obrigkeit verwickelt worden, scheiterte, wurde zum Tode verurteilt und 1707 erschossen $^{3}$ - eine bemerkenswerte Familienparallele! Der Basler Fatio-Zweig, vorwiegend mit Töchtern gesegnet, ist Ende des 18. Jahrhunderts in Basel ausgestorben ${ }^{5}$. Die welschen Fatios oder Fazzy gibt es noch heute in Genf und Lausanne in beträchtlicher Zahl. Eine Rue Louis-Fazzy und der Boulevard James-Fazzy bekunden Verdienste dieses Geschlechtes um die Stadt Genf.

Der Weg des Johannes Fatio zum gesuchten Chirurgus und Geburtshelfernes ist undeutlich. Von seiner Schulzeit wissen wir nichts. Gersters Angabe ${ }^{6}$, Fatio habe sich 1662 an der Basler medizinischen Fakultät immatrikuliert, ist zunächst erstaunlich. Fatio war damals 13jährig! Aber aus den von H.G. Wackernagel 1962 herausgegebenen Basler Matrikeln ${ }^{7}$, geht tatsächlich hervor, daß sich Fatio am 8.X.1662 - 6 Batzen bezahlend - an der Universität immatrikuliert hat. Edgar Bonjour weist ${ }^{8}$ darauf hin, daß in frühen Zeiten der Hochschule, jedenfalls an der propädeutischen facultas artium, schon 12-14jährige mit dem Studium beginnen konnten! Fatio hat sich im Juli 1672 zur Meisterprüfung in der Scherer-Zunft, dem Collegium chirurgicum bei Anwesenheit des Stadtarztes gestellt und die Prüfung bestanden; «nach ausgestandenem Examen vor ein Meuster erkunnt, 
empfangt zugleich die Ehrenzunft». ${ }^{9}$ Voraussetzung war eine dreijährige Lehrzeit des Kandidaten. Bei wem Fatio seine Lehre gemacht hat, ist unbekannt. Dazu kam dann eine Zeit von drei, später von sechs Jahren als Wanderschaftsgeselle. Lange kann Fatio deshalb in Basel nicht studiert haben. Praktisch hätte er an der Fakultät kaum viel lernen können. J.C.Bauhin, Johann Rudolf Burckhardt und Jakob Roth, 1660-1703 nacheinander Inhaber des 3. Lehrstuhles für praktische Medizin ${ }^{10}$, waren «Buchärzte», dozierten aus Büchern; Geburtshilfe und Chirurgie wurden an der Fakultät nicht gelehrt. Chirurgie findet man erst von 1706 an im Lektionskatalog, Geburtshilfe erstmals 1759/6019. In deutschen Landen hatte der Arzt zu Fatios Zeiten noch keinen Zutritt zum Gebärbett. Das besorgten Hebammen, die bis 1725 von den «Hebammenherren», einer Ratskommission, geprüft wurden ${ }^{11}$. Auch für Wundärzte wird es nicht einfach gewesen sein, sich als Geburtshelfer zu betätigen. Woher hatte Fatio die nötigen Kenntnisse? In seinem 1752 posthum erschienenen HebammenLehrbuch: «helvetisch-vernünftige Wehemutter» ${ }^{12}$ - man spürt die beginnende Aufklärung! - kommen sie in erstaunlichem Maße zum Ausdruck. Es scheint gesichert, daß Fatio das entsprechende Manuskript um 1690 ins Reine geschrieben hat. Da heißt es: (§ 152) «seit ich vor nunmehr 18 Jahren hiesigen Landes den Anfang gemacht, den Weibern in schweren Geburten die hilfreiche Hand zu bieten» - also 18 Jahre nach seiner Meisterprüfung von 1672. In Frankreich dagegen war die nicht allein Hebammen überlassene Geburtshilfe schon im 17. Jahrhundert fortgeschritten. Der Pariser Wundarzt François Mauriceau (1637-1709) war ein weitbekannter Geburtshelfer, der 1668 sein Werk: «Des maladies des femmes grosses et accouchées», herausgebracht hat ${ }^{6}$. Es ist zu vermuten, daß Fatio in seiner obligaten Wanderzeit in Paris bei Mauriceau diese Kunst gelernt hat; jedenfalls zitiert er ihn in seiner "Wehemutter» häufig. 1678 hat sich Fatio bei der Basler medizinischen Fakultät um Aufnahme als Aggregatus beworben. Er konnte sich durch einen Doktortitel ausweisen, den er an der Universität von Valence erworben hatte. «Decanum avertit perfectum doctoratum se obtinuisse Valentiae in Delphinatu». ${ }^{13}$ Wann das geschehen ist, kann nicht ausfindig gemacht werden. Die Basler Fakultät hat Fatio abgewiesen: «neque alibi promoti Basilienses a centum annis in facultatem recepti.» Man denkt an das angezweifelte Ferrarenser Doktorat des Paracelsus. Wenig später wird Fatio von der Fakultät gerügt: agyrtarum proprium esse Fazzy

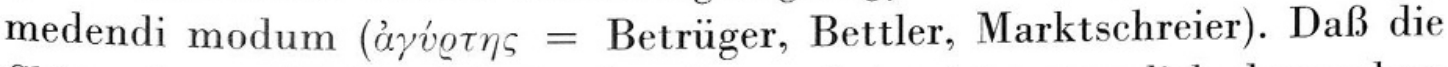
Chirurgie erst 1706 im Lektionskatalog erscheint, ist erstaunlich, denn schon 
1660 hatte ein Doktorand, wohl unter Bauhin, mit der These promoviert: chirurgiae exercitum non esse medico indignum ${ }^{11}$. Ohne die Wundärzte wäre eine medizinische Versorgung der Bevölkerung gar nicht möglich gewesen; die Schererzunft war übrigens zwei Jahrhunderte älter als die Fakultät ${ }^{9}$. Einen Lehrstuhl für Geburtshilfe und Chirurgie, zusammen mit Anatomie, gibt es in Basel erst seit 1818.

Unter welchen Bedingungen Fatio in Valence doktoriert hat, ist nicht zu ermitteln. Es geht aber wohl nicht an, wie das seit Albrecht Burckhardt geschieht ${ }^{10}$, die Universität von Valence als «obskur» zu bezeichnen. Die Hochschule in der Dauphinée ist 1452 vom späteren Louis XI. gegründet worden ${ }^{14}$. Von Anfang an hatte sie vier Fakultäten, auch eine medizinische. Sie hat Höhen und Tiefen erlebt ${ }^{15}$. 1660 ist sie unter Ludwig XIV. reorganisiert und 1689 mit neuen Statuten für die medizinische Fakultät versehen worden. Vermutlich ist Fatio nur kurze Zeit vor 1678 in Valence gewesen, als er sich auf seiner Gesellenwanderschaft befand. Die nötigen Französischkenntnisse mag er sich in Paris erworben haben. Die Universität von Valence ist während der Französischen Revolution eingegangen und zu einer «école centrale supplémentaire» geworden ${ }^{15}$. Grenoble genügte offenbar als Universität für die Dauphinée.

Daß Fatio schon vor seinem Doktorat in Valence auch als Chirurg in Basel geschätzt war, geht schon daraus hervor, daß man bei öffentlichen Anatomien des Ordinarius für Anatomie, Johann Heinrich Glaser (1629-1675), im theatrum anatomicum des unteren Collegiums am Rheinsprung, ihn als «chirurgus magister Facio» zugezogen hat. Der Ordinarius demonstrierte auf der einen Seite der Leiche den anatomischen Befund, und Fatio zeigte auf der anderen chirurgische Eingriffe, ehrerbietig, «detecto capite». ${ }^{10}$ Obschon Basels Professoren und Ärzte den geschickten Chirurgus wiederholt zu Konsultationen holten, galt er in der Ärzteschaft seit seiner Ablehnung eben als ein Agyrta, als Kurpfuscher - auch dies eine Parallele zu Paracelsus! Das dürfte den ehrgeizigen und empfindlichen Mann schwer gekränkt haben und mag seine Teilnahme und seine Führerschaft bei der Aufstandsbewegung gegen die korrupte damalige Basler Obrigkeit im sogenannten «91er Wesen» entscheidend mitbedingt haben.

Fatios geburtshilflich-chirurgisches Können zeigt sich beeindruckend in seiner «helvetischen Wehe-Mutter». Der Autor weist auf seine Absicht hin, «so wenn es Gott gefällt» auch eine von ihm verfaßte Chirurgie zu schreiben ${ }^{12}$. Dazu ist der 42jährige, 1691 als Rädelsführer der fehlgeschlagenen Bürgerrebellion auf dem «Kornmarkt» vor dem Basler Rathaus Enthaup- 
tete, nicht gekommen. Albrecht Burckhardt sagt von Fatio: «er war ein ungewöhnlich heller und selbständiger Kopf, praktisch vortrefflich begabt, auch medizinisch nicht ungebildet; er hätte der Fakultät gut angestanden und hätte die Chirurgie als neues Fach einführen und vertreten können; auch in seiner politischen Tätigkeit hatte er eigentlich das Recht auf seiner Seite».

Über 60 Jahre nach Fatios Tod ist seine «Wehemutter» 1752 in Basel erschienen. Wer das Buch herausgegeben hat, weiß man nicht. In Frage kommen Fakultätsmitglieder - aggregati -, die sich damals wie Johann Staehelin und Joh. Jakob Thurneysen mit Geburtshilfe befaßten; offenbar wagte niemand mit seinem Namen zu dem einst Hingerichteten zu stehen. Julius Gerster hat in seiner Dissertation ${ }^{6}$ als Grund der vielen in der WeheMutter enthaltenen detaillierten Angaben über Patienten, Zeit, Ort und Art die Behandlung überzeugend darlegen können, daß dies Buch dem Manuskript Fatios von 1690 entsprechen muß, abgesehen von einigen hippokratischen Hinzufügungen durch «Buchmediziner». Die vom Herausgeber beigefügten Kupferstich-Tafeln stammen, wie Gerster gezeigt hat, aus Mauriceaus «traité des maladies des femmes grosses». Gerster schreibt: «nur selten findet ein Liebhaber alter medizinischer Werke Fatios Wehe-Mutter und ahnt, welch' ein hervorragender Arzt einst das Buch geschrieben hat». Es ist nicht nur eine verständnisvolle Einführung in die Geburtshilfe für Hebammen: «handelnd von den Weibern insgemein, ihren sonderbaren Geburts- und Leibesteilen, der Empfängnis und den Zeichen derselben; von den Schwangern und den ihnen zustehenden Krankheiten, von der natürlichen und widernatürlichen und schweren Geburt und wie ihnen zu begegnen, von Verpflegung der Sechswöchnerinnen und ihren Ungemachen und Krankheiten». Dazu kommt eine Lehre vom gesunden und kranken Säugling. Darüber hinaus ist das Buch eine Geburtshilfslehre für den Arzt, durch zahlreiche eigene Beobachtungen belegt. Eigene Heilerfolge, wie die Leitung einer Geburt, «da der Gebähr-Mutter Scheide herfürgefallen und reponiert worden war», werden besprochen. Auch an kritischen Bemerkungen fehlt es nicht, etwa: «zu langes Warten der Hebamme nach Ablösung der Nachgeburt - so daß die Frau verblutete». Eindrücklich sind Schilderungen chirurgischer Leistungen, wie die Behebung einer angeborenen Stenose der Harnröhre, oder die operative Trennung von Xiphopagen. Bei solchen Eingriffen hat sich Fatio offenbar häufig vorher mit Medizinern besprochen, aber er - der «Agyrta» war es, der den Eingriff ausführte. Von seinem geburtshilflichen Wirken erklärte er stolz: «mehr als tausend Personen in gefährlichen Kindes-Nöthen, sowohl in Basel als anderen Ortes, nächst 
göttlichen Beystandes geholfen zu haben». Gerster schließt seine Besprechung der Wehemutter etwas affektiv: «er verlangte danach den Armen und Bedrängten zu helfen. So kam es, daß er am Bürgeraufstand von 1691 dessen Führer wurde und für eine gerechte Sache sein Leben hingab.» «Die Volksgunst trug ihn in schwindelnde Höhen und ließ ihn fallen in die tiefste Tiefe» sagt Gustav Steiner ${ }^{9}$.

Das sogenannte «einundneunziger Wesen», eher ein Unwesen, die städtische Revolution von 1691, in der Johannes Fatio, in komplexer Weise dazu motiviert, eine entscheidende, schließlich unglückliche Rolle gespielt hat, ist von der Geschichtsschreibung unterschiedlich beurteilt worden. Erstaunlicherweise erwähnt ihn Gagliardi in seiner «Geschichte der Schweiz» überhaupt nicht ${ }^{16}$. Gustav Steiner ${ }^{9}$ spricht recht hart vom «traurigen Kapitel unserer Geschichte, das schlechtweg nur häßlich und niederträchtig ist». Von Fatio sagt er: «man denkt an Jenatsch mit den starken Gegensätzen und Mischungen eines merkwürdigen Charakters». Fatio war kein großartiger Egmont, als den ihn in schwärmerischer Bewunderung Emma MeyerBrenner $1902^{17}$ in einem larmoyanten Trauerspiel dargestellt hat, sie, die Tochter Karl Brenners, des Basler Vorkämpfers für liberalen Radikalismus. Aber es ist ebenso ungerecht, diesen schwer überblickbaren Menschen mit Eduard Schweizer ${ }^{18}$ als heuchlerischen Demokraten zu disqualifizieren. Eher trifft das Urteil Paul Burckhardts zu ${ }^{19}$, der Fatio als «leidenschaftlich, ehrgeizig und verschlagen» bezeichnet. Er war wohl ein «Mensch in seinem Widerspruch». Daß er in finanzieller Bedrängnis 1679 einen Hypothekarzinsbetrug begangen hat und deswegen vom Rat mit 2 Jahren Stadtverbot bestraft worden ist ${ }^{18}$, mag immerhin gegen seine restlose Integrität sprechen. Das war kurz nach dem kostspieligen Doktortitel in Valence! Steiner erwähnt in diesem Zusammenhang ${ }^{19}$, Fatio sei mit einer Margaretha Schönauer verheiratet gewesen. Von dieser Frau ist sonst nichts zu lesen. Daß es in Basel zu dem über ein halbes Jahr währenden Aufruhr gekommen ist, erstaunt nicht. Es war allerdings kein Volksaufstand, sondern das Rebellieren der in den 15 Zünften und den 3 Kleinbasler Gesellschaften zusammengeschlossenen Bürger. In der damaligen Obrigkeit dominierte der absolutistisch-oligarchisch und korrupt regierende «Kleine Rat». Dazu gehörten die 4 «Häupter», die beiden Bürgermeister und die beiden Oberstzunftmeister - die amtierenden und die pausierenden -, 15 Ratsherren und 15 Zunftmeister - auch in doppelter Zahl - zusammen also 64 «Herren» ${ }^{20}$. Bei der Wahl und Wiederwahl dieses Rates spielten Familieninteressen, Bestechungen mit oft folgendem Meineid, dies nicht getan zu 
haben, eine bedenkliche Rolle. Es ging nicht nur um Ehrenstellen! Beunruhigung in der Bürgerschaft brachten auch der Hüninger Festungsbau durch Vauban ${ }^{18}$ und Bewegungen französischer Truppenteile vor der Stadt. Dazu kam eine Kornteuerung. Das hat zur Revolte beigetragen. Verstimmung brachte auch die Vernachlässigung des großen Rates, der Legislative, in der die Zünfte mit ihren Sechsern vertreten waren; im ganzen ein Gremium von 216 Köpfen! Dieser Rat war -- erstaunlich undemokratisch - in den letzten 60 Jahren nur 5 Mal einberufen worden. So beherrschte eine eigensüchtige «aristokratische» Handelsherren-Oligarchie weniger Familien, in erster Linie der Burckhardt und Socin, die Stadt, unterstützt vom zielstrebigen Mitwirken eines «Weiberregimentes» unter Führung der schlauen und hemmungslosen Frau des Oberstzunftmeisters Christoph Burckhardt ${ }^{18}$, der attraktiven Salomé, geborenen Schönauer. Sie arrangierte ihr zweckmäßig scheinende Bestechungen.

Bei der Besetzung der Ordinariate an der Universität ging es kaum besser zu wie bei den Wahlen in den Rat. Das macht ein Memorial deutlich, mit dem sich der große Mathematiker Jakob Bernoulli - anonym - an eine WahlKontrollkommission - die 4 «Heimlicher» gewandt hatte. Diese Kommission - sie sollte den Meineid ausrotten - war auf Drängen der Bürgerausschüsse eingesetzt worden. Bernoullis Mahnschrift hatte freilich keinen Erfolg ${ }^{11}$.

Wenn Fatio unter dem Einfluß seines ehrgeizigen Vetters Henric Petri, dem Initianten der Bildung der Bürgerausschüsse - 4 aus jeder Zunft - sich schließlich an die Spitze der Opposition gestellt hat, so ist das nach seiner Behandlung durch die medizinische Fakultät nicht unverständlich. Allerdings trieb ihn auch sein Geltungsbedürfnis. Petri war als «Generalprokurator» der Ausschüsse von seinen eigenen Leuten gestürzt worden, weil er sich um die vakante Ratsschreiber-Stelle beworben hatte.

Den Verlauf des 91er Wesens hat neben Peter Ochs ${ }^{21}$ in seiner «Geschichte der Stadt und Landschaft Basel» besonders Eduard Schweizer 1931 erschöpfend dargestellt ${ }^{18}$; manchmal in fast verwirrender Vollständigkeit. Er stützt sich auch auf ein sogenanntes «Basler Manuskript»-1690/91 - der Basler Universitätsbibliothek, das aus dem Notariat Lauterburgers, des Sekretärs der «Ausschüsse», stammt. Aufschlußreich ist auch Gustav Steiners «Ärzte und Wundärzte, Chirurgen-Zunft und Fakultät». ${ }^{9}$ Paul Burckhardt hat die «städtische Revolution von 1691», das Wesentliche festhaltend, in seiner Geschichte der Stadt Basel geschildert ${ }^{19}$. Auf diese Literatur sei hier verwiesen. 
Warum ist der an sich verständliche Bürgeraufstand, wesentlich unter Fatios Führung, gescheitert? Zunächst, es war keine wirkliche Volkserhebung, sondern die Auflehnung oft uneiniger Zunftbrüder unter einer wenig zielklaren Leitung. Eine mitreißende Führung ist weder Petri noch Fatio und seinen Helfern, dem zu braven Johannes Müller und Fatios Schwager, dem Chirurgus Conrad Mosis, geglückt, trotz anfänglicher Erfolge bei erheblichen Zugeständnissen des oft gedemütigten Rates. Gehemmt wurde die Aktion wiederholt durch die von der Tagsatzung zur Schlichtung Mediation - eines als für die Eidgenossenschaft gemeingefährlich betrachteten Geschehens entsandten «Repräsentanten». Am 9. September sind die Herren nach Versuchen zur Schlichtung enttäuscht abgezogen ${ }^{18}$. Sie haben aber die Aufrührer verunsichert und die Obrigkeit zum Eingreifen ermutigt. Hinderlich für das Gelingen der Aktion war auch die schwankende Haltung der Kirche, zumal ihres Antistes Werenfels. Im ganzen ein arges Durcheinander, bei dem es auch zu brutalen Aktionen einzelner Unzufriedener gekommen ist. Fatio und die Seinen sind schließlich der Uneinigkeit der Zunftbürger zum Opfer gefallen. Am 16.8. hatte in Klein-Basel ein Aufstand wider Fatio stattgefunden ${ }^{21}$. Die geplante demokratische Verfassungsform mit 178 «Reformpunkten» hat sich trotz anfänglichen Erfolgen nicht verwirklichen lassen. Klein-Basel und die abgesetzten Ratsherren haben die Gefangensetzung Fatios durchgesetzt. Der wieder zur Macht gekommene Rat hat seine Folterung zugelassen und schließlich den nützlichen Chirurgus und Geburtshelfer zum Tode verurteilt. Der harte Spruch ist am Morgen des 29. September 1691 auf dem Kornmarkt vor dem Rathaus, unter starken Sicherungsmaßnahmen, an Fatio, Müller und Mosis vollstreckt worden. Einen «gerichtlichen Mord» hat das Peter Ochs genannt. Jedenfalls war es ein Akt der Rache der wieder zur Macht Gekommenen. Fatio ist dem Tode in gelassener Haltung entgegengegangen. «Heiße Tränen sah man fließen, als er die Richtbühne betrat». ${ }^{21}$ Henric Petri ist in contumaciam zum Tode verurteilt, sein Bild öffentlich verbrannt worden. 1693 hat er sich, im Ausland in Sicherheit, mit der Schmähschrift «Basel - Babel» an seiner Vaterstadt gerächt.

Als Fazit des mißglückten Versuches einer demokratischen Erneuerung blieb wenig. Immerhin, die Stellung des großen Rates, der Legislative, war gestärkt. Er ist in der Folge, ohne Aufgebot durch den Kleinen Rat, regelmäßig zusammengetreten, übte tatsächlich die höchste Gewalt aus. Die Wahl der «Häupter» gehörte jetzt zu seiner Kompetenz. Der W ahlmodus für Staatsmänner wie für Professoren ist erst 1740 durch ein kompliziertes 
Losverfahren verbessert worden. Eigentliche Bestechungen waren fortan kaum mehr möglich.

Der Zürcher Oberstpfarrer Klingler hat kurz nach 1691 zum Basler Wesen gefunden: «Wie ist es nicht eine Zeit her so mißlich gestanden um die mitverbündete Stadt Basel, in welcher eine landesverderbliche Verwirrung und Aufstand der Unteren gegen die Oberen sich ereignete? Nur, weil man die von Gott empfangene obrigkeitliche Autorität so hoch gesponnen, und weil etliche Wenige allein regieren und das gemeine Gut unter sich allein verteilen wollten». ${ }^{21}$

Über dem Rebellen Fatio darf man den begabten und mutigen Chirurgus und den Geburtshelfer nicht vergessen, den Initiator für dieses Fach an Basels medizinischer Fakultät.

\section{Literatur}

1 Siegfried, Paul, Basler Straßennamen, Helbing \& Lichtenhahn, Basel 1921.

${ }^{2}$ Roth, Paul, Die Straßennamen der Stadt Basel. Helbing \& Lichtenhahn, Basel 1959.

${ }^{3}$ Historisch-biographisches Lexikon der Schweiz, Bd. III, Neuenburg 1926.

${ }^{4}$ Huber-Burckhardt, Albert, Die Familie Fatio. Basler Nachrichten, Sonntagsblatt 1913, Nrn. 28 und 29.

${ }^{5}$ Lutz, Markus, Baslerisches Bürgerbuch. Schweighauser, Basel 1819.

${ }^{6}$ Gerster, Julius, Johannes Fatio, ein Basler Chirurg und Geburtshelfer des XVII. Jahrhunderts. Diss. Basel, Benno Schwabe, Basel 1917.

7 Wackernagel, Hans Georg, Matrikel der Universität Basel, Bd. III. Universitäts-Bibliothek Basel 1962.

${ }^{8}$ Bonjour, Edgar, Die Universität Basel 1460-1960. Helbing \& Lichtenhahn, Basel 1960.

${ }^{9}$ Steiner, Gustav, Ärzte, Wundärzte, Chirurgenzunft und medizinische Fakultät in Basel. Basler Jahrbuch 1954, S. 179.

${ }^{10}$ Burckhardt, Albrecht, Geschichte der medizinischen Fakultät 1460-1900. Reinhardt, Basel 1917.

11 Staehelin, Andreas, Geschichte der Universität Basel 1662-1818. Helbing \& Lichtenhahn, Basel 1957.

${ }^{12}$ Fatio, Johannes, Helvetisch-vernünftige Wehe-Mutter. Joh. Rud. Imhof, Basel 1752.

${ }^{13}$ Decreta medica, Univ. Archiv. Staatsarchiv Basel 1571-1804.

${ }^{14}$ La grande Encyclopédie, Tome 31. H. Lamirault et Cie., Paris 1885.

15 Nadel, Abbé, Histoire de l'université de Valence. Valence 1861.

${ }^{16}$ Gagliardi, Ernst, Geschichte der Schweiz, Bd.2, 3. Auflage. Orell Füßli, Zürich 1938.

${ }^{17}$ Meyer-Brenner, Emma, Johann Fatio, Trauerspiel. Birkhäuser, Basel 1902.

${ }^{18}$ Schweizer, Eduard, Eine Revolution im alten Basel. 109. Neujahrsblatt. Basel 1931.

${ }^{19}$ Burckhardt Paul, Geschichte der Stadt Basel. 2. Aufl., Helbing \& Lichtenhahn, Basel 1957. 
${ }^{20}$ Burckhardt, August, Bürgerschaft und Regiment im alten Basel. 97. Neujahrsblatt. Basel 1919.

${ }^{21}$ Ochs, Peter, Geschichte der Stadt und Landschaft Basel, Bd.7. Schweighauser, Basel 1831.

\section{Zusammenfassung}

Nach Darstellung des Schicksals der ursprünglich aus dem Val d'Ossola stammenden, 1635 mit einem Zweig in Basel eingebürgerten Familie Fatio, oder Fazzy, wird das Leben des 1649 in Basel geborenen Johannes Fatio geschildert. Nach einer gründlichen Ausbildung hat dieser fähige, ehrgeizige Mann 1672 als Wundarzt - Chirurgus - die Meisterprüfung in der Schererzunft bestanden; wenig später ist er in Valence zum Dr. med. promoviert worden. Die Anerkennung dieses Titels hat die Basler medizinische Fakultät abgelehnt; Fatio galt unbilligerweise bei den Akademikern als Kurpfuscher - «Agyrta». Er aber hat während vieler Jahre trotz seiner Verbitterung in Basel als geschickter und gesuchter Chirurg, namentlich auch als Geburtshelfer, gewirkt. Posthum ist sein hervorragendes Hebammenlehrbuch «helvetisch-vernünftige Wehe-Mutter» erschienen. Der von der Fakultät so schlecht behandelte Fatio hat sich im sogenannten «91er Wesen» - bald als Anführer - dem Bürgeraufstand rebellierender Zünfte gegen eine korrupte, oligarchische Obrigkeit angeschlossen. Nach anfänglichen Reorganisationserfolgen ist die gelegentlich brutale Revolte, hauptsächlich wegen der Uneinigkeit der Aufständischen, gescheitert. Fatio ist im September 1691 gefangengesetzt und mit zweien seiner Mitrebellen auf dem Kornmarkt vor dem Rathaus hingerichtet worden.

\section{Summary}

One branch of the family of Fatio or Fazzy moved from the Valley of Ossola to Basel and was granted citizenship in 1635. John Fatio, an able and ambitious man, born of this family in 1649 , passed the examination as a Master-Surgeon at the Guild of the Barbers after a thorough apprenticeship. Shortly afterwards, he acquired the M.D. in Valence. But the University of Basel refused to acknowledge this title. Hence, the academic establishment took Fatio for a scharlatan ("agyrta"). Fatio, skillfull and sought-for by his patients, practised nevertheless as surgeon and obstetrician for many years. Only after his death, his excellent textbook for midwives "Helvetisch-vernünftige Wehe-Mutter" was published.

Fatio also engaged himself politically and became leader of a rebellion (91er Wesen) against the corruption of the City Governement. The rebellion succeeded in having some reform brought by, but was soon put down due to internal differences. Fatio was captured in 1691 and executed in front of the City Hall along with two of his companions.

Prof. Dr. med. Friedrich Rintelen

Bruderholzrain 55

CH-4059 Basel 